\title{
New Passenger Services in Railway Stations Based on Mode Group Diversity Multiplexing Optical Fiber Communications
}

\author{
Dafa Loum $^{1,2,3}$, Charles Tatkeu, ${ }^{1,2}$, Iyad Dayoub ${ }^{1,3}$, Marc Heddebaut ${ }^{1,2}$ and Jean Michel Rouvaen ${ }^{1,3}$ \\ 1. Univ. Lille Nord de France- F-59000 Lille, France \\ 2. IFSTTAR, LEOST, F-59666 Villeneuve d'Ascq, France \\ 3. IEMN, DOAE, University of Valenciennes and Hainaut Cambrésis, F-59313 Valenciennes Cedex, France
}

\begin{abstract}
Nowadays, because of its wide bandwidth and high communication capability, the optical fiber is more and more used for high data rate transmission of information in railway environments. Conventionally, only one service is sent over the fiber at a time. However, many different services can be simultaneously conveyed in railway stations such as passenger information service, cellular phone, Wi-Fi... The objective of the work proposed in this paper is to demonstrate the potential benefits of transmitting radio signals over fiber in a railway environment. The main idea is to exploit the full capacity of the fiber by transmitting multiple services using the same fiber. Since, different services are operating in different frequency bands; we propose a new multiplexing technique called Mode Group Diversity Multiplexing (MGDM) to ensure the transmission of multiple services using the same fiber, without additional infrastructure. There are numerous advantages of the proposed technique, e.g., faster and reliable data exchange, high resolution video surveillance capability, high data rate information exchange in railway stations. We present, in this paper, the physical characteristics of optical fibers, performance of MGDM multiplexing technique, and the influence of the laser excitation conditions at the entrance of the fiber on the performances of the system.
\end{abstract}

Key words: Optical fiber, passenger services, railway station, mode group diversity multiplexing.

\section{Introduction}

The development of cable networks with optical fibers has an impact on a large number of applications in the civil and military domains. Optical fibers are known for their large bandwidth and their high data rate, superior to those offered by other technologies. However, this bandwidth is not yet fully utilized by the currently available techniques. Generally a single service is transmitted by fiber, while several services could be combined within the same optical fiber. At present, single-mode (SMF-Single-Mode Fiber) and multi-mode (MMF-Multi-Mode Fiber) optical fibers equip more and more buildings, in particular,

Corresponding author: Marc Heddebaut, $\mathrm{PhD}$, senior researcher, research fields: telecommunications, EMC, signal processing. E-mail: marc.heddebaut@ifsttar.fr. shopping malls, places and sites of public transport sites such as stations, airports, highways, ports or multimodal platforms. In these places, fibers connections are often used as means of extension of radio systems in particular between buildings. Furthermore, $90 \%$ of the new buildings in Europe are at present equipped with multi-modes optical fibers MMF [1]. To enable multiple services, (multi-frequency bands operation), we propose the exploitation of a new multiplexing technique called MGDM - Mode Group Diversity Multiplexing, which is a technique based on the transmission and the spatial reception of data. It consists of using 2 or 3 laser sources simultaneously inside the same fiber. The main objective is to provide multiple services using the same fiber, without additional infrastructure. In this paper we present, in the first part, the characteristics of various 
types of optical fibers, before highlighting the advantages of the MGDM multiplexing technique. We also study the influence of the axial delay (or offset) and of the spot size at the entrance of the fiber. We evaluate the transmission performance in terms of communication Bit Error Rate (BER). This evaluation study is performed by simulation using the "VPI ${ }^{\mathrm{TM}}$ Systems" tools. We operate at three conventional wavelengths commonly used for radio transmission over fiber (850 nm, $1300 \mathrm{~nm}$ and $1550 \mathrm{~nm}$ ). We consider fiber optics lengths from $10 \mathrm{~m}$ to $3 \mathrm{~km}$. After several simulations, we show that the optimal "spot size” is around $8 \mu \mathrm{m}$ and that up to three different services can be implemented efficiently in a single optical fiber. Considering these different services, we then discuss the 1-D and 2-D OCDMA codes used for the multiplexing techniques. In this paper, we concentrate on the 2-D codes which are associated to the MGDM technique. Finally, we conclude and give directions for future research.

\section{Characteristics of Optical Fibers}

Optical fiber is constituted of a very fine glass or plastic thread which possesses the property of guiding light. It serves the transmissions of ground and oceanic

Table 1 Characteristics of various types of optical fibers.

\begin{tabular}{|c|c|c|c|c|}
\hline Type of optical fiber & \multicolumn{2}{|c|}{ Single-mode } & \multicolumn{2}{|c|}{ Multi-mode } \\
\hline Nature & Graded-index fiber & Step-index fiber & Graded-index fiber & Step-index fiber \\
\hline Diameter core/cladding & $10 \mu \mathrm{m} / 125 \mu \mathrm{m}$ & $50-62.5 \mu \mathrm{m} / 125 \mu \mathrm{m}$ & $200 \mu \mathrm{m} / 300 \mu \mathrm{m}$ \\
\hline Bandwidth & $10 \mathrm{GHz} / \mathrm{km}$ & $100-1000 \mathrm{MHz} / \mathrm{km}$ & $25 \mathrm{MHz} / \mathrm{km}$ \\
\hline Wavelength/Frequency & $1300 \mathrm{~nm} / 230.7 \mathrm{THz} 1550 \mathrm{~m} / 193.5 \mathrm{~Hz}$ & \multicolumn{2}{|c|}{$850 \mathrm{~nm} / 352.9 \mathrm{THz} 1300 \mathrm{~nm} / 230.7 \mathrm{THz}$} \\
\hline
\end{tabular}

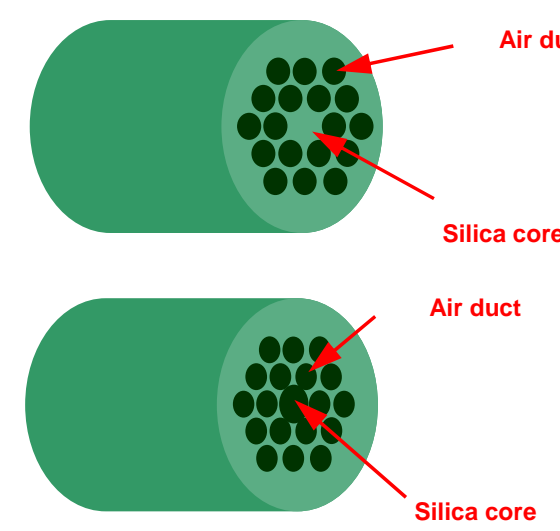

Fig. 1 Example of a micro-structured optical fiber.

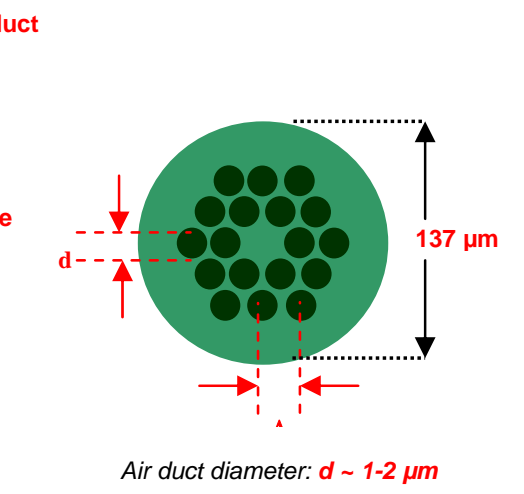

Air duct diameter: $d \sim 1-2 \mu m$ It supports a "wide band" network through which can be carried out various types of services (television, telephony, video conferencing...). There are various types of optical fiber: single-mode, multi-mode and micro-structured fiber.

The optical fiber presents many advantages: it offers wide bandwidth of the Terahertz order, and enables data rates of several gigabits per second. It offers a high level of safety during the transmissions, with low attenuations. It also has a long lasting life, which requires few interventions according to maintenance. It provides a thermal insensitivity, a high degree of immunity against electromagnetic radiations and jamming. However, optical fiber possesses some disadvantages such as difficulties of adaptation with the opto-electronic transducers during installation. It requires precise mechanical alignment and specially adapted connectors. Moreover, the initial installation cost is high compared to conventional networks and it is hard to find adequately trained personnel. Table 1 presents characteristics of various types of optical fibers and Fig. 1 presents an example of a micro-structured optical fiber. 
In spite of the high level of performance, signals which propagate across the optical fiber undergo attenuation. Various losses like the absorption, the modal dispersal, the imperfection of the connections, the presence of the curvatures and the micro-curvatures inside the fiber are usually represented by Rayleigh distributions. The attenuation of the signal depends essentially on the nature of the fiber. The single-mode fiber at wavelength $1550 \mathrm{~nm}$ has attenuation of about $0.2 \mathrm{~dB} / \mathrm{km}$, at $1300 \mathrm{~nm}$ attenuation is about $0.4 \mathrm{~dB} / \mathrm{km}$ and the multi-mode fiber of wavelength at $850 \mathrm{~nm}$ has attenuation of about $3 \mathrm{~dB} / \mathrm{km}$.

In Table 2, we present the advantages and disadvantages of various types of optical fiber: single-mode, multi-mode and micro-structured.

Having presented the characteristics of various types of fibers, in the following paragraph, we develop the multi-users services aspects intended for railway stations applications. For this purpose, we present and evaluate the performances of multiplexing techniques capable of implementing multiple access and managing multi-users.

\section{Mode Group Diversity Multiplexing (MGDM)}

The Mode Group Diversity Multiplexing (MGDM) technique is based on the spatial transmission and reception of data [3]. It consists of sending packet information corresponding to various users. It is an optical technique which aims at creating independent communication channels on a multi-mode optical fiber, using subsets of propagating modes. By associating a service with a propagated mode, the MGDM can offer same features as multiplexing using wavelength with a lower cost. This technique is depicted in following Fig. 2. At one extremity of the optical fiber, several laser beams noted " $\mathrm{e}_{\mathrm{i}}$ " carrying different passenger services, feed the dielectric waveguide. They are characterized by a spot size radius, noted "w", and an offset radius in the plane $\mathrm{xOy}$. At the opposite extremity, the energy corresponding to these different laser beams is concentrated in disks of radius " $\mathrm{r}_{\mathrm{i}}$ " where the different passenger services signals can be simply recovered.

To efficiently manage MGDM multiplexing technique, the generation of certain number of optical codes and the choice of multi-mode fiber is necessary. This approach provides several distinct, independent propagation channels in the optical fiber. Associated with these different propagation channels, we can also encode the digital signals on each of these channels.

\section{Different Optical Codes Used}

The principle of data encoding not only ensures secure transmission, but also allows several users or services to communicate on the same transmission medium.

There are several types of optical codes, including the 1-D, 2-D and 3-D codes. Among the 1-D codes there are OOC-Optical Orthogonal Code, PC-Prime Code and EPC-Extended Prime Code.

In the following paragraphs, we explain how these codes are constructed and generated.

\subsection{OOC Codes}

OOC codes are composed of unipolar sequences characterized by four parameters $\left(\mathrm{L}, \mathrm{W}, h_{a}, h_{c}\right)$ where $\mathrm{L}$ is the length of the sequence, $\mathrm{W}$ is the weight of the code corresponding to the number of chips equal to " 1 ", $h_{a}$ and $h_{c}$ are respectively the constraints for autocorrelation and cross-correlation.

The formula providing the available number of users is: $N_{o o c} \leq\left\lfloor\frac{L-1}{W(W-1)}\right\rfloor[1]$.

Table 3 provides, for example, an OOC code of $\mathrm{N}=5$ users, length of code $\mathrm{L}=71$ and weight of code $\mathrm{W}=4$.

The Fig. 3 gives position of chips equal to 1 , the autocorrelation and the cross-correlation of OOC code.

Maximum autocorrelation is the weight of the code and the cross-correlation is between 0 and 1 . 
Table 2 Comparison of the various types of optical fiber: advantages and disadvantages.

\begin{tabular}{|c|c|c|}
\hline Types of optical fiber & Advantages & Disadvantages \\
\hline Single-mode & $\begin{array}{c}\text { Very large bandwidth. } \\
\text { No distortion. }\end{array}$ & Very high price \\
\hline Multi-mode with step-index & $\begin{array}{c}\text { Low price } \\
\text { Ease of implementation }\end{array}$ & $\begin{array}{c}\text { Heasonable bandwidth loss and distortion of the signal } \\
\text { Good quality of transmission }\end{array}$ \\
\hline Multi-mode with graded-index & $\begin{array}{c}\text { Large bandwidth } \\
\text { Low distortion }\end{array}$ & $\begin{array}{c}\text { Difficulties of manufacturing. } \\
\text { Limited development. }\end{array}$ \\
\hline Micro-structured &
\end{tabular}

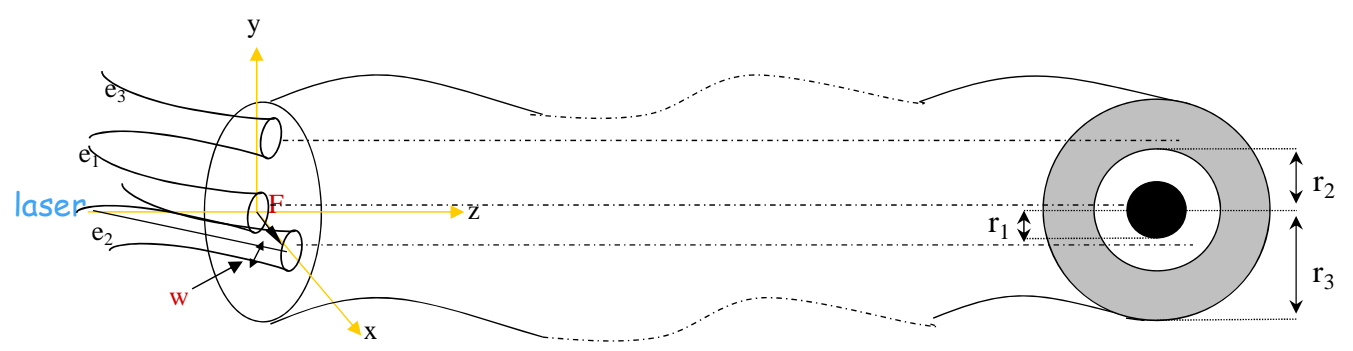

Fig. 2 Mode Group Diversity Multiplexing (MGDM) technique implementation.

Table 3 Example generation of OOC codes for 5 users.

\begin{tabular}{|c|c|c|c|c|c|c|c|}
\hline \multirow{6}{*}{ OOC code } & $N$ & $L$ & $W$ & \multicolumn{4}{|c|}{ Position of chips equal to 1} \\
\hline & \multirow{5}{*}{5} & \multirow{5}{*}{71} & \multirow{5}{*}{4} & 1 & 2 & 33 & 49 \\
\hline & & & & 1 & 5 & 51 & 58 \\
\hline & & & & 1 & 16 & 17 & 59 \\
\hline & & & & 1 & 20 & 61 & 65 \\
\hline & & & & 1 & 6 & 28 & 44 \\
\hline
\end{tabular}

\subsection{PC Codes}

The generation of PC codes performed using Galois's formula GF (p) of a prime number p such as:

$$
\begin{aligned}
& S_{i}^{p}(j)=\left\{S_{i 0}, S_{i 1}, \ldots, S_{i(p-1)}\right\} \\
& \text { with:i et } j \in G F(p), G F(p)=\{0,1, \ldots, p-1\} \\
& \quad S_{i j}=\{i . j\}(\bmod p)
\end{aligned}
$$

The following formula is used:

$$
C_{i}^{p}(n)= \begin{cases}1 & \text { if } n=S_{i}^{p}(j)+j p \quad i, j \in G F(p) \\ 0 & \text { otherwise }\end{cases}
$$

Using equations (2) and (3) we present in Table 4 an example of PC code. For $\mathrm{N}=5$ users we obtain a code of length $L=25$, with weight of code $W=5$.

In Fig. 4, for the PC code, maximum autocorrelation is the weight of the code but autocorrelation values are duplicated. The cross-correlation is situated between 0 and 1.

\subsection{2-D Codes}

The 2D codes enable the spreading of the data in both domains simultaneously, which allows to decrease the constraints on the length of the codes $(\mathrm{L}$, $\mathrm{F}$ ), on the number of available codes $\mathrm{N}$ as well as improving the performances of the system in term of Bit Error Rate (BER) for a given number of active users. Fig. 5 presents an example of a $2 \mathrm{D}$ system in both time and frequency domains.

A family of 2D codes used simultaneously in the temporal and spectral domains is characterized by the parameters ( $\mathrm{LxF}, \mathrm{W}, h_{a}, h_{c}$ ), where $\mathrm{W}$ is the weight of the matrices of code, $h_{a}$ and $h_{c}$ are the values of autocorrelation and cross-correlation defined by:

$$
h_{a}=\max _{\tau}\left(\sum_{i=0}^{L-1} \sum_{j=0}^{F-1} c_{i}^{j} * c_{i}^{j \oplus \tau}\right) h_{c}=\max _{\tau}\left(\sum_{i=0}^{L-1} \sum_{j=0}^{F-1} c_{i}^{j} * d_{i}^{j \oplus \tau}\right)
$$


Table 4 Example generation of PC code for 5 users.

\begin{tabular}{|c|c|c|c|c|c|}
\hline$N$ & $L=p^{2}$ & $W=p$ & $\mathrm{i}$ & $S_{i j}$ & PC-Prime Code \\
\hline \multirow{3}{*}{5} & & & 0 & 00000 & 1000010000100001000010000 \\
\cline { 4 - 5 } & \multirow{3}{*}{5} & \multirow{3}{*}{5} & 1 & 01234 & 1000001000001000001000001 \\
\cline { 3 - 5 } & & & 2 & 02413 & 1000000100000010100000010 \\
\cline { 3 - 5 } & & & 4 & 03142 & 1000000010010000000100100 \\
\hline
\end{tabular}

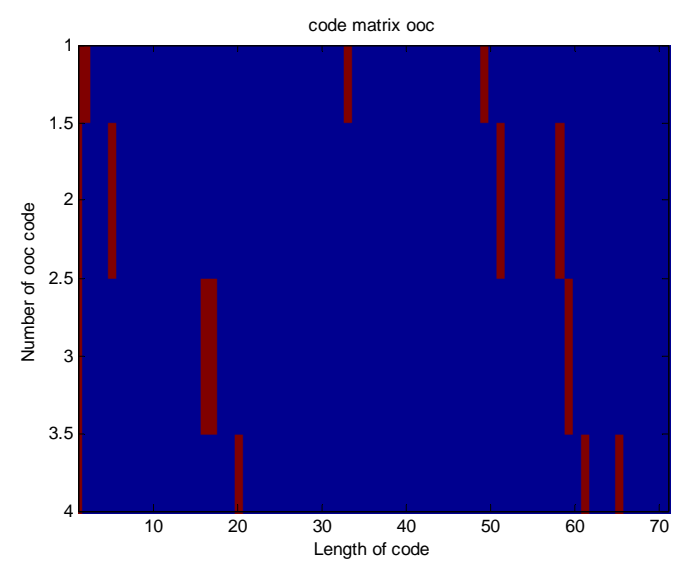

(a) Positions of chips to 1 of OOC code

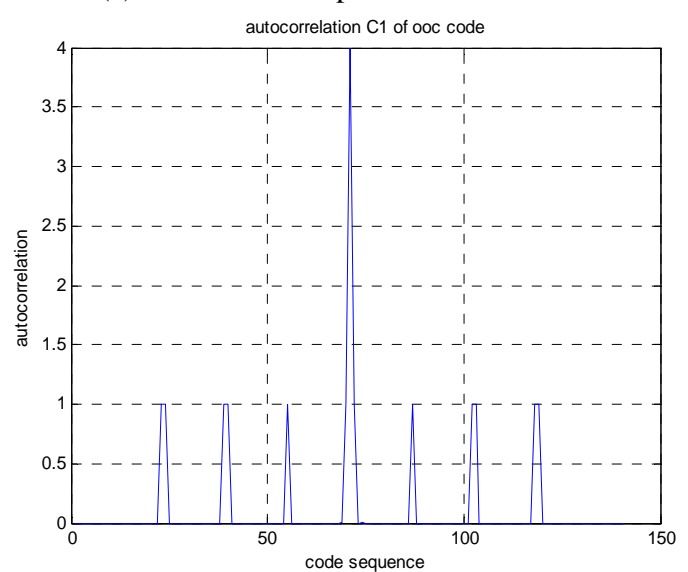

(b) Autocorrelation of OOC code

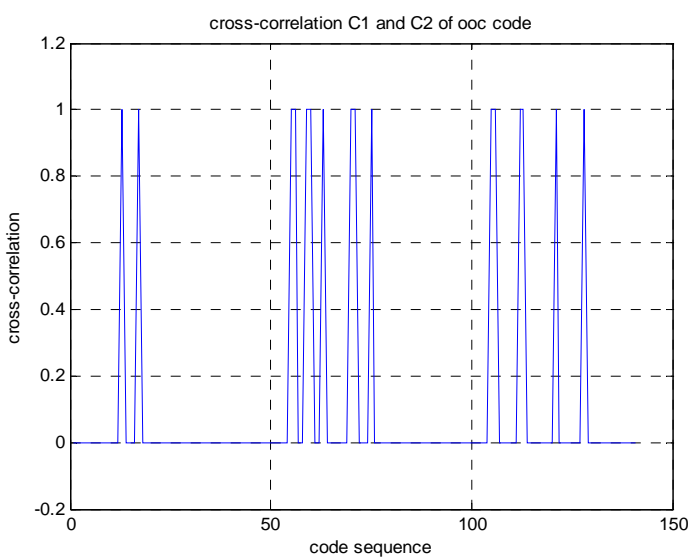

(c) Cross-correlation of OOC code

Fig. 3 (a) Position of chips, (b) autocorrelation and (c) cross-correlation of OOC code.

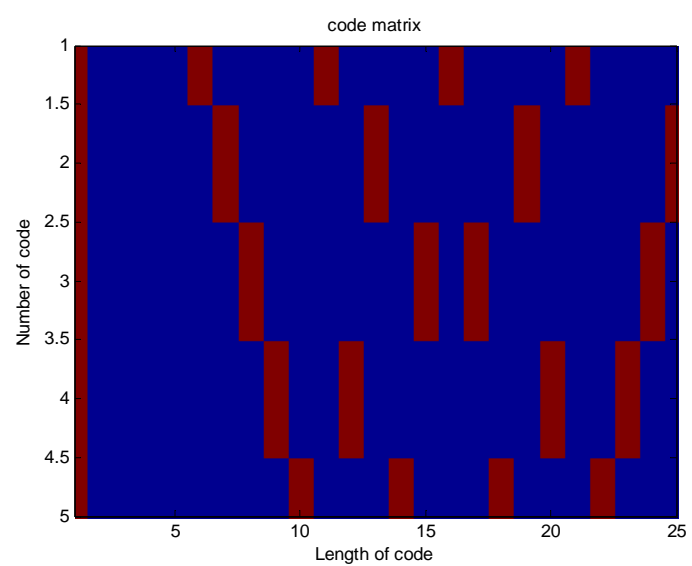

(a) Positions of chips to 1 of PC code

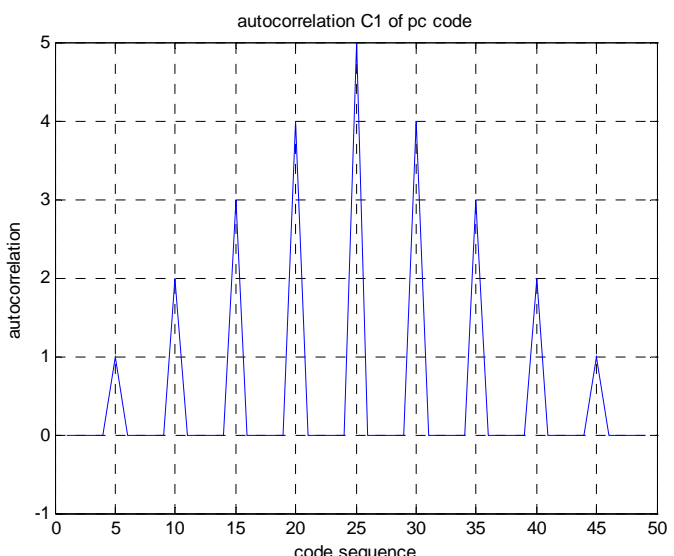

(b) Autocorrelation of PC code

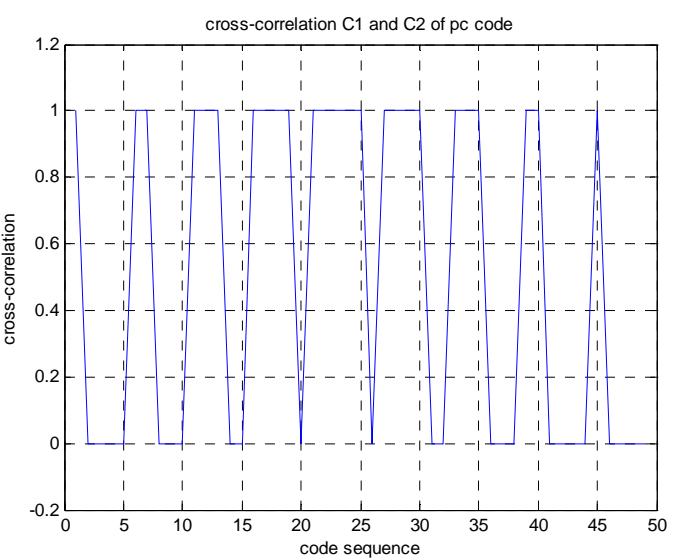

(c) Cross-correlation of PC code

Fig. 4 (a) Position of chips, (b) autocorrelation and (c) cross-correlation of PC code. 


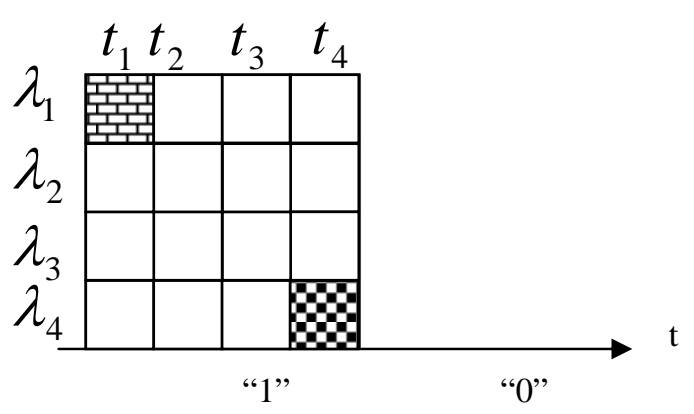

Fig. 5 2-D OCDMA, time and wavelength.

with: $c$ and $d$ are two matrices of a family of code, $c_{i}^{j}$ and $d_{i}^{j} \in[0,1], i \in[0, L-1], j \in[0, F-1] \tau$ represent the asynchronous gap between two codes, $\tau \in[0, F-1]$ et $\oplus$ is a sum modulo- $F$.

The objective is to generate families of codes which allow to obtain a large number of sequences with optimal properties of autocorrelation and cross-correlation $\left(h_{a}=h_{c}=1\right)$ in order to limit the impact of Multiple Access Interference - IAM.

\subsection{Example of FFH Code}

The following Fig. 6 presents the position of chips and the evolution of autocorrelation and cross-correlation functions of 2-D code FFH-Fast Frequency Hopping.

We have represented in Table 5 an example of FFH code. We obtain, for $\mathrm{N}=20$ users, a code of length $\mathrm{L}=$ 25 and weight of code $\mathrm{W}=5$.

The autocorrelation of FFH code is best because we obtain a single peak corresponding to the maximum of correlation. The values of cross-correlation are between 0 and 1 . We conclude that 2D codes are quite interesting to use because they increase the number of available users.

Table 5 Example generation of FFH code for 20 users.

\begin{tabular}{|c|c|c|c|}
\hline \multirow{2}{*}{ FFH code } & $N=p(p-1)$ & $L=p^{2}$ & $W$ \\
\cline { 2 - 4 } & 20 & 25 & 5 \\
\hline
\end{tabular}

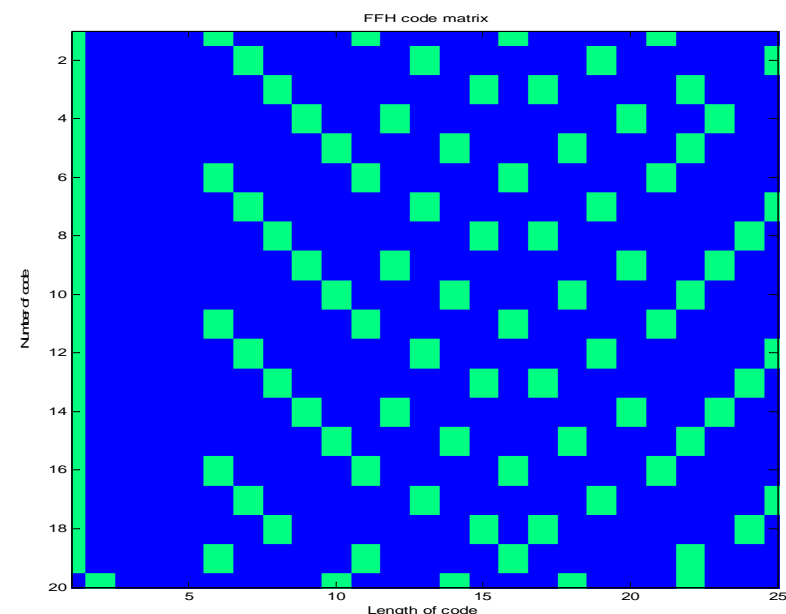

(a) Positions of chips to 1 of FFH code

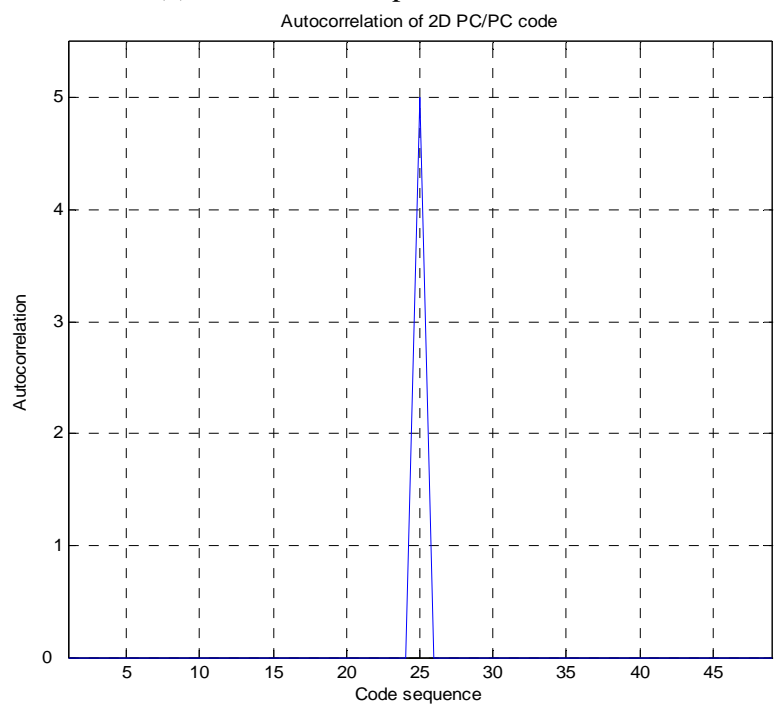

(b) Autocorrelation of FFH code

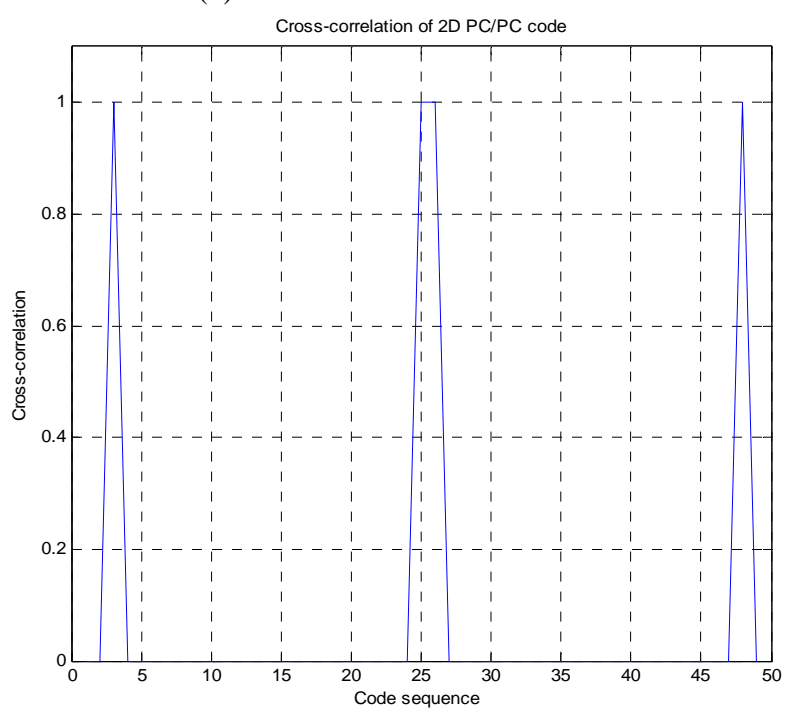

(c) Cross-correlation of FFH code

Fig. 6 (a) Position of chips, (b) autocorrelation and (c) cross-correlation of FFH code. 


\section{Simulations Results}

The following simulation results are obtained by using the $\mathrm{VPI}^{\mathrm{TM}}$ systems software. VPI ${ }^{\mathrm{TM}}$ Systems is a simulation software for optical transmission channel, which can be used to develop and support sophisticated design tools for photonic devices including network components.

It supports modeling several types of optical fiber by using several opto-electronic components. In our case, it takes into account the majority of the optical fiber parameters.

We study the evolution of the Bit Error Rate (BER) with respect to the emitted power for three typical wavelengths used for radio transmission over fiber (850 nm, $1300 \mathrm{~nm}$ and $1550 \mathrm{~nm}$ ), by using fiber lengths ranging from $10 \mathrm{~m}$ to $3 \mathrm{~km}$ and for different "spot size" of $3 \mu \mathrm{m}$ and $8 \mu \mathrm{m}$.

In Fig. 7 we have an example of a simulated channel of optical transmission with wavelength of $850 \mathrm{~nm}$ and spot sizes $3 \mu \mathrm{m}$ and $8 \mu \mathrm{m}$.

It is better to use fiber length lower than $1 \mathrm{~km}$ because the gain obtained is about $7 \mathrm{~dB}$ compared to optical fiber of $3 \mathrm{~km}$. This type of "domestic" application could be used inside railway stations.

The variation of spot size permits to define the number of laser source that could be sent according to the diameter of optical fiber. In practical it is possible to send simultaneously up to 3 laser sources inside the same core of optical fiber and to manage up to 3 different channels. These allow transmission of much more information at the same time.

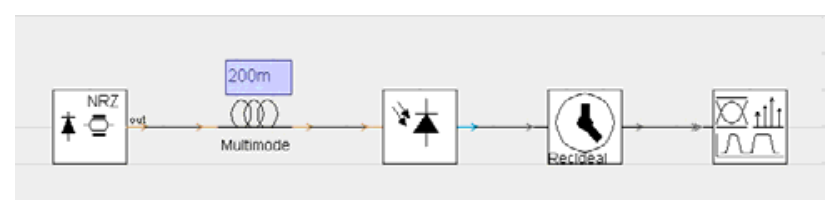

Fig. 7 Example channel of optical transmission with wavelength $850 \mathrm{~nm}$.

\section{Conclusion and Perspectives}

We presented the physical characteristics of different types of optical fibers, namely SMF, MMF and micro-structured fibers. We develop the MGDM technique for the multiple users and we give some examples of optical codes necessary for this technique. We presented the results of the simulations obtained using VPI ${ }^{\mathrm{TM}}$ Systems software. These results lead us to conclude that the best configurations are obtained for spot size of $8 \mu \mathrm{m}$. With a spot size of $8 \mu \mathrm{m}$, up to 3 lasers sources may be sent. This shows the potential benefits of transmitting signals over fiber in a railway environment. In the future work we will include a third dimension (3-D codes) in simulation to increase the capacity or number of users that could be used.

\section{References}

[1] I. Dayoub, A. Zaouche, J. M. Rouvaen, C. Lethien, J. P. Vilcot and D. Decoster, Wireless Systems Radio-optic demonstrator for distributed antenna system indoor wireless applications using low-cost VCSELs, European Transactions on Telecommunications 18 (7) (2007) 811-814.

[2] J. A. Saheli, Code division multiple-access techniques in optical fiber networks-Part II, IEEE Transactions on Communications 37 (8) (1989) 824-842.

[3] M. Awad, I. Dayoub, J. M. Rouvaen and J. P. Vilcot, RoF \& multi services in single-MMF LAN using mode group diversity multiplexing, invited paper to ICTTA'08, April, Damas.

[4] D. Loum, Transmissions radio haut débit sur fibres optiques appliquées aux transports terrestres, Rapport d'avancement de thèse: décembre 2008-décembre 2009, INRETS-LEOST-09-739-FR. 